\title{
A case of intra cardiac yolk sac tumour
}

\author{
J V Amalaseelan ${ }^{1}$, D C Pieris ${ }^{1}$ and M Munasinghe ${ }^{2}$
}

(Index words: yolk sac tumour, malignant intracardiac tumour)

\section{Introduction}

Germ cell tumours (GCTs) are benign or malignant neoplasms arising from primordial germ cells which can be gonadal or extragonadal in origin. In contrast to adult patients, the incidence of extragonadal tumours exceeds gonadal ones in patients younger than 15 years old [1]. Heart is an extremely rare site for a GCT and the outcome is not as good as other extra gonadal GCTs due to its lethal cardiac complications.

\section{Case report}

This 5-year old girl with insignificant past medical history was incidentally found to have a cardiac murmur by her general practitioner. She was referred to a cardiologist for further evaluation and the diagnosis of osteum secundum atrial septal defect (OSASD) was made. At that stage her condition was amenable to device closure and she was given a date for the procedure in 3 months time.

When she came for the device closure 3 months later, another cardiac assessment was done as a routine preoperative procedure. Interestingly, it showed an intracardiac tumour, which was not seen in the previous echocardiograms. It involved the interatrial and interventricular septa and a provisional diagnosis of "atypical" atrial myxoma was made. Within 24 hours the patient was taken up for an open surgery. On cardiopulmonary bypass a right atrial approach was made and a large tumour was identified deeply infiltrating into the interatrial septum, interventricular septum and septal leaflet of tricuspid valve. The tumour was completely excised macroscopically and ASD and iatrogenic VSD were closed with a pericardial patch and a polytetrafluroethylene patch respectively. Her post-operative period was complicated with complete heart block and she recovered well with temporary cardiac pacing.

The histological examination of the specimen showed a pure yolk sac tumour arising from endocardium with focal infiltration of myocardium (Figures 1 and 2). The child was referred to the National Cancer Institute (NCI) for further management. Pre-operative tumour markers were not done as the possibility of GCT was not thought of. At NCI post-operative $\alpha$-fetoprotein (AFP) level was $4917 \mathrm{ng} / \mathrm{ml}$ (less than $10 \mathrm{ng} / \mathrm{ml}$, normally) and $\beta$-hCG level was less than $2 \mathrm{mIU} / \mathrm{ml}$ (normal). Metastatic screening with CT of chest and abdomen were negative.
She was started with a chemotherapeutic regime according to MAKEI 89 protocol [2] which consists of cisplatin, etoposide and ifosphamide. After the third cycle her $\alpha$-fetoprotein level became normal $(1.9 \mathrm{ng} / \mathrm{ml})$. A total of 6 cycles of chemotherapy were given. Her chemotherapy course was complicated with febrile neutropaenia and intercurrent candidal infection which were treated with antibiotics and amphotericin respectively. After the completion of drug therapy a permanent pace maker was implanted. One year after the diagnosis she is well with a good exercise tolerance.

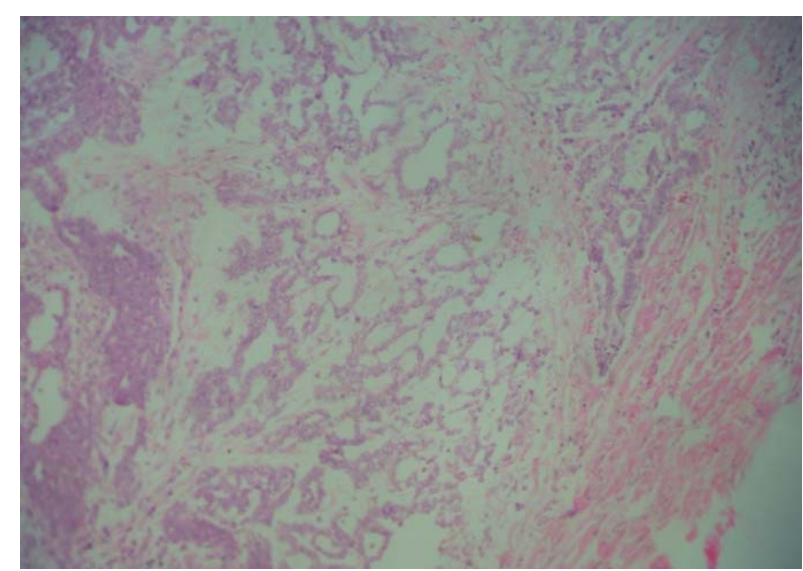

Figure 1. Reticular pattern formed by the loose network of flat/cuboidal cells. Infiltration into cardiac muscles also noted $(H \& E \times 100)$.

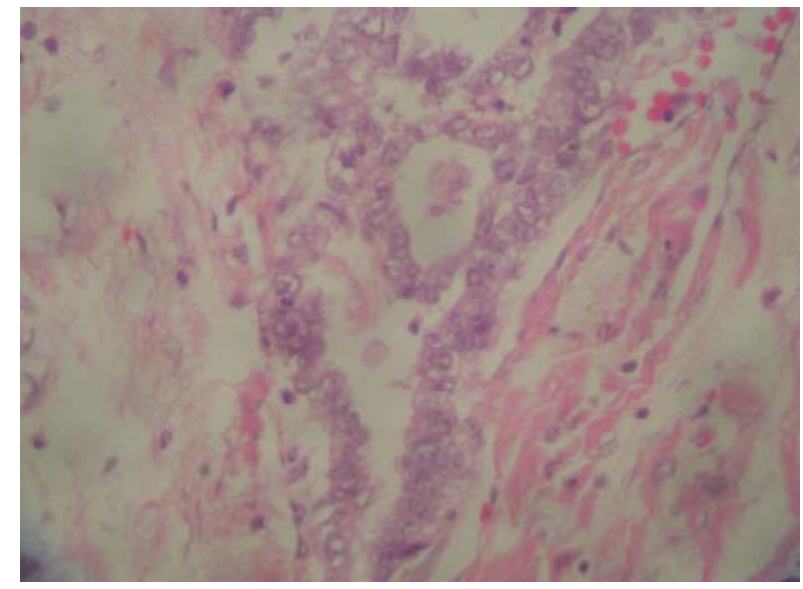

Figure 2. Characteristic Schiller Duvall body is seen in the centre. Tumour infiltration into the cardiac muscle also revealed (H\&E $\times 400)$.

${ }^{1}$ National Cancer Institute of Sri Lanka, Maharagama, and 'Lady Ridgeway Hospital, Colombo, Sri Lanka.

Correspondence: JVA, e-mail <Julan_24@yahoo.com>. Received 7 August and accepted 19 September 2009. Competing interests: none declared. 


\section{Discussion}

Sacrococcygeal region, mediastinum, retroperitoneum and other pelvic organs are the common sites for a primary extragonadal GCT in paediatric age group [1]. GCTs have many histological types according to cellular differentiation and grade. Myocardium is an extremely rare site of origin for an extra gonadal GCT and only 3 cases have been reported. All previously documented tumours are also yolk sac or predominantly yolk sac in origin $[3,4,5]$. Our patient differs from the previously reported cases in that the tumour was arising from the endocardium and focally infiltrating the myocardium rather than originating in the myocardium itself.

Even though these tumours are highly treatable, management of intracardiac tumours are often complicated by the cardiac problems such as cardiac failure, tamponade or arrhythmias. The echocardiographic appearance of an intracardiac tumour within a period of 3 months in itself hints at its malignant potential. Therefore, an open surgery was performed to excise the tumour and to close the ASD. Because of the deep infiltration of the tumour into the inter-atrial and ventricular septa, an extensive surgery was performed to maximally debulk the tumour knowing it could damage the conductive pathway.

The tumour marker, AFP was extremely helpful in assessing the tumour response to chemotherapy. Complete response to chemotherapy was indicated by persistent normal level of AFP after the chemotherapy. She will be followed up for her life time to monitor the side effects of the drugs and tumour recurrence. The management of these highly treatable cardiac tumours demands a strong multidisciplinary approach to deal with unanticipated complications of the tumour and the treatment.

\section{References}

1. Ries LA, Smith MA, Gurney JG, et al. eds. Cancer incidence and survival among children and adolescents: United States SEER Program 1975-1995. Bethesda, Md: National Cancer Institute. 1999: 125-35.

2. Göbel U, Bamberg M, Engert J, et al. Treatment of nontesticular germ cell tumors in children and adolescents with BEP and VIP: initial results of the MAKEI 89 therapy study. Klinische Padiatrie 1991; 203: 236-45.

3. Melinda JM, Richard AH, William SF, James WZ. Intracardiac yolk sac tumor and dysrhythmia as an etiology of pediatric syncope. Pediatrics 2004; 113: 374-6.

4. Graf M, Blaeker H, Schnabel P, et al. Intracardiac yolk sac tumor in an infant girl. Pathology, Research and Practice 1999; 195:193-7.

5. Parvathy U, Balakrishnan KR, Ranjith MS, et al. Primary intracardiac yolk sac tumor. Pediatric Cardiology 1998; 19: 495-7. 